Publicacions Matemàtiques, Vol 34 (1990), 181-197.

\title{
FOURIER ANALYSIS OF A SPACE OF HILBERT-SCHMIDT OPERATORS- NEW HA-PLITZ TYPE OPERATORS
}

\author{
JAAK PEETRE
}

\begin{abstract}
If a group acts via unitary operators on a Hilbert space of functions then this group action extends in an obvious way to the space of HilbertSchmidt operators over the given Hilbert space. Even if the action on functions is irreducible, the action on H.S. operators need not be irreducible. It is often of considerable interest to find out what the irreducible constituents are. Such an attitude has recently been advocated in the theory of "Ha-plitz" (Hankel + Toeplitz) operators. In this paper we solve this problem the space of H.S. operators over the Hilbert space $L^{2}\left(\Delta, \mu_{\alpha}\right)$ of square integrable functions over the unit disk $\Delta$ equipped with the Dzhrbashyan measure $d \mu_{\alpha}(z)=(\alpha+1)\left(1-|z|^{2}\right)^{\alpha} d A(z)(\alpha>-1)$. This complements the earlier results. In particular we discover many new Ha-plitz type operators. The question of their smoothness properties $\left(S_{p}\right.$ estimates etc.) is however only touched upon.
\end{abstract}

\section{Introduction}

If a group acts via unitary operators on a Hilbert space of functions then this group action extends in an obvious way to the space of Hilbert-Schmidt (H.S.) operators over the given Hilbert space. (More generally, one can consider H.S. operators from one Hilbert space into another with a different group action on each of these spaces.) Even if the action on functions is irreducible, the action on H.S. operators need not be irreducible. It is often of considerable interest to find out what the irreducible constituents are. Such an attitude has recently been advocated in the theory of "Ha-plitz" (Hankel + Toeplitz) operators.

Example 1. Consider the spaces $A=A^{\alpha, 2}(\Delta)$ (definition in Sec. 1), $A^{1}$ $=$ the orthogonal complement of $A$ in $L^{2}\left(\Delta, \mu_{\alpha}\right)$ (definition in Sec. 1), $\bar{A}=$ the space consisting of all conjugates of the functions in $A$. Operators from $A$ into $\bar{A}$ have been studied from this point of view in [JP1] ("small" Hankel operators of higher weight). Similarly, operators from $A$ into $A^{\perp}$ have been studied in [BJP] ("big" dittos). In both cases the group is the projective group of conformal selfmaps of the unit disk $\Delta$ in $\mathrm{C}$ or a double cover. 
Example 2. The study of the case when $\Delta$ is replaced by the unit ball in $\mathbf{C}^{n}$ has been initiated in [P1].

Example 3. H.S. operators on $L^{2}\left(\mathbf{R}^{n}\right)$ under the action of the " $a x+b^{n}$-group (dilations + translations) are analyzed in [JP2], [P2] (so-cailed "paracommutators").

In this paper we want to decompose the space of H.S. operators over $L^{2}\left(\Delta, \mu_{\alpha}\right)$. This complements the results of [JPI], [BJP]. In particular, we discover many new Ha-plitz type operators. The question of their smoothness properties $\left(S_{p}\right.$ estimates etc.) is however only touched upon.

There are good hopes that one could be able to play the same game with other symmetric domains (in higher dimensions), in the first place the ball (cf. [P1]).

The organization of the paper is as follows.

Sec. 1 introduces the notation.

In Sec. 2 we consider various invariant meastres on the space of H.S. operators over $L^{2}\left(\Delta, \mu_{\alpha}\right)$. In particular, this allows to write the space in question as a tensor product where one factor is the space $L^{2}(\hat{G}), \hat{G}$ being the group of all conformal selfmaps of $\Delta$. The results of this Section are of independent interest and we intend to return to them on a subsequent occasion.

In Sec. 3 we then apply Plancherel's theorem in the latter space to produce. the desired decomposition of $L^{2}\left(\Delta, \mu_{\alpha}\right)$.

Sec. 4 is devoted to some examples showing how known type of (big) Hankel operators [JPI], [BJP] (Example 1 ultra) fit into our new scheme.

In Sec. 5, returning to the general case, we write down the expression for the H.S.-norm of our new Hankel operators.

Sec. 6 briefly touches upon the $S_{p}$-theory, still in its embryo.

In Sec. 7 we sketch an alternative infinitesimal approach to our problem. It is not entirely successful, as we have not been able to carry out the spectral analysis of the Casimir operator encountered:

In Sec. 8 we specialize this to the case of operators from $A^{\alpha, 2}(\Delta)$ into itself (generalized Toeplitz operators).

Finally, there is an appendix to which we have deferred some (dull?) calculations pertaining to Sec. 2 . We intend to apply this result in a different context in a subsequent publication.

Acknowledgment. I am greatful to the referee for his many comments, the scholarly ones and also the humoristic ones. He also detected a missing factor $1 /\left(16 \pi^{2}\right)$ in formula (6), Sec. 2. In addition, I am obliged to Richard Askey and André Unterberger for precious information.

\section{Mainly notation}

Let us thus begin by fixing some notation: 
$\Delta$ - unit disk in the complex plane $\mathbf{C}$.

$\mathbf{T}$ - its boundary (the unit circumference).

$d A(z)=\frac{d x d y}{\pi}=\frac{i d z d \bar{z}}{2 \pi}-$ normalized Euclidean area measure on $\Delta$.

$d I(z)=\frac{d A(z)}{\left(1-|z|^{2}\right)^{2}}-$ invariant (Poincaré) measure on $\Delta$.

$d \mu_{\alpha}(z)=(\alpha+1)\left(1-|z|^{2}\right)^{\alpha} d A(z)(\alpha>-1)$ - Dzhrbashyan measure.

$L^{2}\left(\Delta, \mu_{\alpha}\right)=$ the space of square integrable functions with respect to $d \mu_{\alpha}$.

$A^{\alpha, 2}(\Delta)=\operatorname{Hol}(\Delta) \cap L^{2}\left(\Delta, \mu_{\alpha}\right)$ - Dzhrbashyan (or weighted Bergman) space, the space of holomorphic functions in $L^{2}\left(\Delta, \mu_{\alpha}\right) .{ }^{1}$ The corresponding norm and the corresponding inner product will be written $\|\cdot\|_{\alpha}$ and $(\cdot, \cdot)_{\alpha}$ respectively.

The space of H.S. operators on $L^{2}\left(\Delta, \mu_{\alpha}\right)$ can be identified with $L^{2}\left(\Delta, \mu_{\alpha}\right) \otimes$ $L^{2}\left(\Delta, \mu_{\alpha}\right)=L^{2}\left(\Delta \times \Delta, \mu_{\alpha}\right)$, that is, a H.S. operator $T=T_{F}$ on $L^{2}\left(\Delta, \mu_{\alpha}\right)$ is given by a kernel $F\left(z_{1}, z_{2}\right)$ such that

$$
\left\|T_{F}\right\|^{2}=\int_{\Delta \times \Delta}\left|F\left(z_{1}, z_{2}\right)\right|^{2} d \mu_{\alpha}\left(z_{1}\right) d \mu_{\alpha}\left(z_{2}\right)<\infty .
$$

$G=S U(1,1)-$ Möbius group.

$\hat{G}=P S U(1,1)=S U(1,1) / \pm 1$ - projective group; the elements $x$ of $\hat{G}$ are thus fractional linear functions of the type $x(z)=\frac{a z+b}{c z+d}$, where $\left(\begin{array}{ll}a & b \\ c & d\end{array}\right)$ is in $G$, uniquely determined by $x$ up to sign.

$\tilde{G}$ - universal covering group of $G$ or $\hat{G}$.

It will be necessary to distinguish carefully between these groups. An element of $G$ is an element $x$ of $\hat{G}$ plus a choice of a determination of the square root $\sqrt{x^{\prime}}$. Similarly, an element of $\tilde{G}$ is an element of $\hat{G}$ plus a choice of a determination of the logarithm $\log x^{\prime}$ (then we can define arbitrary powers of $x^{\prime}$ ).

$d H(x)$ denotes the Haar measure on any of these groups.

The group $\bar{G}$ acts on elements $f(z)$ of $L^{2}\left(\Delta, \mu_{\alpha}\right)$ or $A^{\alpha, 2}(\Delta)$ according to the rule

$$
f(z) \mapsto f(x(z))\left(x^{\prime}(z)\right)^{\frac{a+2}{2}}\left(=f\left(\frac{a z+b}{c z+d}\right)(c z+d)^{-(a+2)}\right) \quad(x \in \tilde{G}) .
$$

That these group actions are unitary follows from the formula

$$
d \mu_{\alpha}(x(z))=\left|x^{\prime}(z)\right|^{\alpha+2} d \mu_{\alpha}(z) .
$$

In [JPR], P. 63 this space was called the Bargmann-Bergman-Besov-Dahrbashyan-FisherFock-Segal space. As the referee suggests, one could as well have included the names Petersson and Hecke too. The reason why we have given preference to the name Dahrbashyan here is that we think that hitherto one has not in the literature payed sufficient tribute to the achievements of the Armenian school of analysis. 
Similarly, a kernel $F\left(z_{1}, z_{2}\right)$ experiences the change

$$
F\left(z_{1}, z_{2}\right) \mapsto F\left(x\left(z_{1}\right), x\left(z_{2}\right)\right)\left(x^{\prime}\left(z_{1}\right)\right)^{\frac{\alpha+^{2}}{2}}\left(\overline{x^{\prime}\left(z_{2}\right)}\right)^{\frac{\alpha+^{2}}{2}} \quad(x \in \tilde{G}) .
$$

Our main concern is thus to decompose the space $S_{2}\left(L^{2}\left(\Delta, \mu_{\alpha}\right)\right)$ of H.S. operators under the last action.

Begin by writing ${ }^{2}$

$$
F\left(z_{1}, z_{2}\right)=K_{\alpha}\left(z_{1}, z_{2}\right) F_{0}\left(z_{1}, z_{2}\right)
$$

where $K_{\alpha}\left(z_{1}, z_{2}\right)=\left(1-z_{1} \bar{z}_{2}\right)^{-(\alpha+2)}$ is the reprodcing kernel in $A^{\alpha, 2}(\Delta)$. Then $F_{0}$ transforms as

$$
F_{0}\left(z_{1}, z_{2}\right) \mapsto F_{0}\left(x\left(z_{1}\right), x\left(z_{2}\right)\right) \quad(x \in \hat{G} \operatorname{sic} !)
$$

this in view of the well-known formula

$$
K_{\alpha}\left(x\left(z_{1}\right), x\left(z_{2}\right)\right)\left(x^{\prime}\left(z_{1}\right)\right)^{\frac{\alpha+2}{2}}\left(\overline{x^{i}\left(z_{2}\right)}\right)^{\frac{\alpha+2}{2}}=K_{\alpha}\left(z_{1}, z_{2}\right) \quad(x \in \tilde{G}) .
$$

We agree to refer to $F_{0}$ as the "reduced" kernel. Thus we may work with $F_{0}$ instead of $F$. In terms of $F_{0}$ the H.S. norm is given by

$$
\left\|T_{F}\right\|^{2}=\int_{\Delta \times \Delta}\left(\frac{\left(1-\left|z_{1}\right|^{2}\right)\left(1-\left|z_{2}\right|^{2}\right)}{\left|1-z_{1} \bar{z}_{2}\right|^{2}}\right)^{\alpha+2}\left|F_{0}\left(z_{1}, z_{2}\right)\right|^{2} d I\left(z_{1}\right) d I\left(z_{2}\right) .
$$

Its invariant character is thus apparent.

\section{Invariant measures}

Clearly

$$
d J=d I \otimes d I
$$

is an invariant measure on $\Delta \times \Delta$. But, as the group $\hat{G}$ does not act transitively on this set, it is not unique. There are other interesting invariant measures on $\Delta x \Delta$ or rather on $\Delta x \Delta \backslash$ (diagonal), the manifold of all ordered pairs of elements of $\Delta$ (or oriented hyperbolic segments).

${ }^{2}$ One motivation for this construction comes from Berezin's program for quantization (cf. [P3]). The referee comments that "the idea of factorization of automorphic forms goes back to many, many people, probably much before Berezin (Poincaré, for example)". 
Let thus an ordered pair $\left(z_{1}, z_{2}\right)$, with $z_{1} \in \Delta, z_{2} \in \Delta$, be given. Consider the unique (oriented) geodesic through $z_{1}$ and $z_{2}$, directed from $z_{2}$ to $z_{1}$.

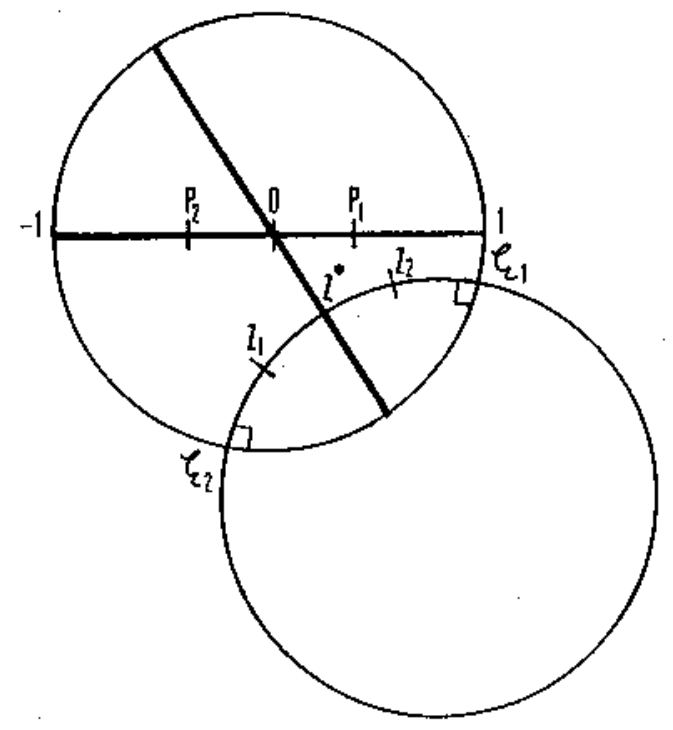

There is a unique transformation $\xi$ in $\hat{G}$ such that its preimage is the interval $(-1,1)$ and such that the preimages of $z_{1}$ and $z_{2}$ are two symmetrically situated (about the origin 0 ) points $p_{1}$ and $p_{2}$ on $(0,1)$ and $(-1,0)$ respectively:

$$
z_{1}=\xi\left(p_{1}\right), z_{2}=\xi\left(p_{2}\right) \quad\left(1>p_{1}=-p_{2}>0\right) .
$$

Let $\lambda$ denote the exponential of the hyperbolic distance between $z_{1}$ and $z_{2}$ (i.e. $\log \lambda=\int_{p_{1}}^{p_{2}} \frac{d x}{1-x^{2}}=\frac{1}{2} \log \frac{1+p_{1}}{1-p_{1}}-\frac{1}{2} \log \frac{1+p_{2}}{1-p_{2}}=\log \frac{1+p_{1}}{1-p_{1}}$ or $\left.\lambda=\frac{1+p_{1}}{1-p_{1}}\right)$. Thus the pair $\left(z_{1}, z_{2}\right)$ is uniquely determined by the pair $(\xi, \lambda)$, and the manifold of all ordered pairs gets identified with the product $\hat{G} \times \mathbf{R}_{+}$. Notice that $\hat{G}$ acts on the first factor via multiplication from the left (see (4)) and trivially on the second factor (the hyperbolic distance is preserved), i.e. to $\left(x\left(z_{1}\right), x\left(z_{2}\right)\right)$, where $x \in \hat{G}$, there corresponds the pair $(x \xi, \lambda)$. It follows that an invariant measure on the set $\Delta x \Delta \backslash$ (diagonal) is given by

$$
d J^{\prime}=d H(\xi) \cdot \frac{d \lambda}{\lambda}
$$

We can further identify the group $\hat{G}$ itself with the manifold of geodesics equipped with a base point. Let $\zeta_{1}$ and $\zeta_{2}$ be the endpoints of the geodesic on 
T and let $m$ be the exponential of the hyperbolic distance from the base point to the Euclidean midpoint $z^{*}$ of the geodesic (= arc of a Euclidean orthogonal circle). We may regard $\left(\zeta_{1}, \zeta_{2}, m\right)$ as coordinates for the corresponding group element $\xi$. Then the Haar measure on $\hat{G}$ can be written

$$
d H(\xi)=\frac{\left|d \zeta_{1}\right| \cdot\left|d \zeta_{2}\right|}{\left|\zeta_{1}-\zeta_{2}\right|^{2}} \cdot \frac{d m}{m}
$$

Let further $m_{1}$ and $m_{2}$ measure the distance from $z_{1}$ and $z_{2}$ to $z^{*}$. Then (5) can be rewritten as

$$
d J^{\prime}=\frac{\left|d \zeta_{1}\right| \cdot\left|d \zeta_{2}\right|}{\left|\zeta_{1}-\zeta_{2}\right|^{2}} \cdot \frac{d m_{1}}{m_{1}} \frac{d m_{2}}{m_{2}}
$$

A computation, which I defer to an appendix, reveals that the Radon-Nikodym derivative of the measure (3) with respect to the one in $(5)$ or $\left(5^{\prime}\right)$ is given by

$$
\frac{d J}{d J^{\prime}}=\frac{1}{16 \pi^{2}} \frac{m_{1}^{2}-m_{2}^{2}}{m_{1} m_{2}} .\left(=\frac{1}{8 \pi^{2}}\left(\lambda-\lambda^{-1}\right)\right) .
$$

(A priori one can say that it must be a homogeneous function of degree 0 in $m_{1}$ and $m_{2}$.) If we put $\lambda=e^{\sigma}$, where the quantity $\sigma$ thus has the interpretation of hyperbolic distance, then we have in (6) essentially the hyperbolic $\operatorname{sine}, \sinh \sigma$, which perhaps looks more convincing.

\section{Plancherel theorem}

For the group $\hat{G}$ this theorem tells us that under left multiplication by group elements (left regular representation) the Hilbert space $L^{2}(\hat{G})$ decomposes into an orthogonal sum

$$
L^{2}(\hat{G})=\sum_{m=1}^{\infty} \oplus D_{m} \oplus \sum_{m=1}^{\infty} \oplus \bar{D}_{m} \oplus C \oplus \bar{C}
$$

(the case of $G$ is in $[L]$ from which the present case $\hat{G}$ easily follows]. It consists thus of a "discrete" part and a "continuous" part. Here I will focus on the discrete part only, taking each summand separately. As $D_{m}$ and $\vec{D}_{m}$ are each others conjugates it suffices to consider $D_{m}$. It is known that $D_{m}$ is isometrically isomorphic to $A^{\beta, 2}(\Delta) \otimes \overline{A^{\beta, 2}(\Delta)}$ where $\beta=2 m-2$. Notice that (in contrast to $G$ ) for the group $G$ only even degrees occur! Thus $D_{m}$ is not irreducible but contains $A^{\beta, 2}(\Delta)$ with infinite multiplicity.

In the following discussion it is useful to bear in ones mind the following purely algebraic fact - essentially a consequence of Schur's lemma (cf. [JPW]). 
Lemma. Let $V$ be any simple module (over any $\mathrm{C}$-algebra) and consider an irreducible submodule $W$ of the direct sum $V \oplus V \oplus V \oplus \ldots$ (with elements $\left.x_{1} \oplus x_{2} \oplus \ldots\right)$. Then the elements of $W$ are of the form $p_{1} x \oplus p_{2} x \oplus \ldots$ where $x \in V$ runs through this module and $p_{1}, p_{2}, \ldots$ are fixed complex numbers.

The general form of an element of $D_{m}$ is

$$
f(\xi)=\int_{\Delta} \Phi(\xi(t), \bar{t})\left(\xi^{\prime}(t)\right)^{m} d \mu_{\beta}(t)
$$

where $\Phi$ thus is analytic in the first argument and anti-analytic in the second argument. In particular, $D_{m}$ contains any function of the form

$$
f(\xi)=\int_{\Delta} \phi(\xi(t)) \overline{\psi(t)}\left(\xi^{\prime}(t)\right)^{m} d \mu_{\beta}(t)
$$

with $\phi, \psi \in A^{\beta, 2}(\Delta)$ (cf. [L], p. 181). If we fix $\psi \neq 0$ and let $\phi$ vary we get a submodule of $D_{m}$ isomorphic to $A^{\beta, 2}(\Delta)$. Choosing an orthonormal basis $\left\{\psi_{j}\right\}$ in $A^{\beta, 2}(\Delta)$ (e.g. the standard basis obtained by normalizing the monomials $\left.\left\{z^{j}\right\}\right)$ we decompose $D_{m}$ into a direct sum of irreducible submodules. Next pick an orthonormal basis $\left\{B_{i}\right\}$ also in the space $L^{2}\left((1, \infty), \Lambda_{\alpha}\right)$, where

$$
16 \pi^{2} \cdot d \Lambda_{\alpha}(\lambda)=\left(\frac{2 \lambda}{\lambda^{2}+1}\right)^{2(\alpha+2)}\left(\lambda-\lambda^{-1}\right) \frac{d \lambda}{\lambda}
$$

(Notice that

$$
\left.\frac{\left(1-\left|z_{1}\right|^{2}\right)\left(1-\left|z_{2}\right|^{2}\right)}{\left|1-z_{1} \bar{z}_{2}\right|^{2}}=\left(\frac{2 \lambda}{\lambda^{2}+1}\right)^{2} .\right)
$$

Then for every H.S. operator $T_{F}$ the corresponding reduced kernel $F_{0}$ (see Sec. 1) admits the decomposition

$$
F_{0}\left(z_{1}, z_{2}\right)=\sum_{i} A_{i}(\xi) B_{i}(\lambda)
$$

Let us restrict our attention to the case when all the $A_{i}$ belong to $D_{m}$, that is, they are of the form

$$
A_{i}(\xi(t))=\int_{\Delta} \sum_{j} \phi_{i j}(\xi(t)) \overline{\psi_{j}(t)}\left(\xi^{\prime}(t)\right)^{m} d \mu_{\beta}(t)
$$

with $\psi_{j}(t) \in A^{\beta, 2}(\Delta)$. We then obtain

$$
F_{0}\left(z_{1}, z_{2}\right)=\int_{\Delta} \sum_{i, j} \phi_{i j}(\xi(t)) \overline{\psi_{j}(t)}\left(\xi^{\prime}(t)\right)^{m} d \mu_{\beta}(t) B_{i}(\lambda)
$$


Invoking the lemma (or rather the general philosophy behind it) we see that the most general irreducible submodule is gotten by taking all the $\phi_{i j}$ proportional to one and the same function $\phi$ in $A^{\beta, 2}(\Delta)$, that is, $\phi_{i j}=p_{i j} \phi$ for suitable scalar $p_{i j}$. Such an irreducible submodule is thts spanned by reduced kernels of the form

$$
F_{0}\left(z_{1}, z_{2}\right)=\int_{\Delta} \phi(\xi(t)) a(t, \lambda)\left(\xi^{\prime}(t)\right)^{m} d \mu_{\beta}(t)
$$

with

$$
a(t, \lambda)=\sum_{i, j} p_{i j} \overline{\psi_{j}(t)} B_{i}(\lambda)
$$

By change of variable (see (1)) this formula can be written

$$
F_{0}\left(z_{1}, z_{2}\right)=\int_{\Delta} \phi(t) a\left(\xi^{-1}(t), \lambda\right)\left(\overline{\left(\xi^{-1}\right)^{\prime}(t)}\right)^{m} d \mu_{\beta}(t),
$$

that is,

$$
F_{0}\left(z_{1}, z_{2}\right)=\int_{\Delta} \phi(t) A_{0}\left(z_{1}, z_{2}, t\right) d \mu_{\beta}(t)
$$

where

$$
A_{0}\left(z_{1}, z_{2}, t\right)=a\left(\xi^{-1}(t), \lambda\right)\left(\overline{\left(\xi^{-1}\right)^{t}(t)}\right)^{m} .
$$

Reintroducing the reproducing kernel we can finally write the kernel itself as

$$
F\left(z_{1}, z_{2}\right)=\int_{\Delta} \phi(t) A\left(z_{1}, z_{2}, t\right) d \mu_{\beta}(t)
$$

with

$$
A\left(z_{1}, z_{2}, t\right)=K_{\alpha}\left(z_{1}, z_{2}\right) A_{0}\left(z_{1}, z_{2}, t\right) .
$$

Notice that the kernel $A$ obeys the transformation law (cf. (2))

$$
A\left(x\left(z_{1}\right), x\left(z_{2}\right), x(t)\right)=A\left(z_{1}, z_{2}, t\right)\left(x^{\prime}\left(z_{1}\right)\right)^{\frac{a+2}{2}}\left(\overline{x^{\prime}\left(z_{2}\right)}\right)^{\frac{\alpha+2}{2}}\left(\overline{x^{\prime}(t)}\right)^{-m} \text {; }
$$

similarly for $A_{0}$ :

$$
A_{0}\left(x\left(z_{1}\right), x\left(z_{2}\right), x(t)\right)=A_{0}\left(z_{1}, z_{2}, t\right)\left(\overline{x^{i}(t)}\right)^{-m} .
$$

Direct check that such a kernel defines an invariant submodule:

$$
\begin{gathered}
F_{0}\left(x\left(z_{1}\right), x\left(z_{2}\right)\right)=\int_{\Delta} \phi(t) A_{0}\left(x\left(z_{1}\right), x\left(z_{2}\right), t\right) d \mu_{\beta}(t) \\
=\int_{\Delta} \phi(x(t)) A_{0}\left(x\left(z_{1}\right), x\left(z_{2}\right), x(t)\right) d \mu_{\beta}(x(t)) \\
=\int_{\Delta} \phi(x(t))\left(x^{\prime}(t)\right)^{m} A_{0}\left(z_{1}, z_{2}, t\right)\left|x^{\prime}(t)\right|^{-2 m} d \mu_{\beta}(x(t)) \\
=\int_{\Delta} \phi(x(t))\left(x^{\prime}(t)\right)^{m} A_{0}\left(z_{1}, z_{2}, t\right) d \mu_{\beta}(t),
\end{gathered}
$$

where we in the last step once again involved (1).

Remark. In hindsight we could perhaps have written down this formula right at the beginning, but we find it more instructive to follow the path of discovery. 


\section{Examples}

Example 1. $m=1$. Conventional big Hankel operators [A], [AFP] correspond to the kernel

$$
F\left(z_{1}, z_{2}\right)=K_{\alpha}\left(z_{1}, z_{2}\right) \int_{z_{1}}^{z_{2}} \phi(\zeta) d \zeta
$$

(If we introduce the primitive $b$ of $\phi$ (i.e. $b^{\prime}=\phi$ ) we get the usual expression

$$
\left.F\left(z_{1}, z_{2}\right)=K_{\alpha}\left(z_{1}, z_{2}\right)\left(b\left(z_{1}\right)-b\left(z_{2}\right)\right)\right)
$$

Check of the covariance:

$$
F_{0}\left(x\left(z_{1}\right), x\left(z_{2}\right)\right)=\int_{x\left(z_{2}\right)}^{x\left(z_{1}\right)} \phi(t) d t=\int_{z_{2}}^{z_{1}} \phi(x(t)) x^{\prime}(t) d t .
$$

But this is not of the form (7). It is however easy to rewrite the formula in the form (7). Indeed, one has to take

$$
\begin{aligned}
& A\left(z_{1}, z_{2}, t\right)=\int_{z_{2}}^{z_{1}} \frac{d \zeta}{(1-\zeta \bar{t})^{2}}=\left[\frac{1}{\bar{t}(1-\zeta \bar{t})}\right]_{z_{1}}^{z_{2}} \\
= & \frac{1}{\bar{t}}\left(\frac{1}{1-z_{1} \bar{t}}-\frac{1}{1-z_{2} \bar{t}}\right)=\frac{z_{1}-z_{2}}{\left(1-z_{1} \bar{t}\right)\left(1-z_{2} \bar{t}\right)} .
\end{aligned}
$$

Example 2. $m=2$. We have the (big) "Lagrange-Hankel" operator [BIP], [Pi] corresponding to

$$
F_{0}\left(z_{1}, z_{2}\right)=b^{\prime}\left(z_{1}\right)+b^{\prime}\left(z_{2}\right)-2 \frac{b\left(z_{1}\right)-b\left(z_{2}\right)}{z_{1}-z_{2}} .
$$

To get the transition to our present form of notation we invoke the third order derivative $b^{\prime \prime \prime}=\phi$ of the given $b$. (This is in accordance with the requirement of "Boil's lemma" [GP].) Then we find

$$
F_{0}\left(z_{1}, z_{2}\right)=\int_{x\left(z_{2}\right)}^{x\left\{z_{1}\right)} \frac{\left(z_{1}-\zeta\right)\left(z_{2}-\zeta\right)}{z_{1}-z_{2}} \phi(\zeta) d \zeta .
$$

As in Example 1 one can write down the corresponding kernel $A_{0}$,

$$
A_{0}\left(z_{1}, z_{2}\right)=\frac{z_{1}-z_{2}}{\left(1-z_{1} \bar{t}\right)^{2}\left(1-z_{2} \bar{t}\right)^{2}} .
$$

The method in [P1] is however preferable, and applicable also for $m>2$. 


\section{H.S.-norm}

In the preceding discussion we have lost track of the H.S.-norm. It is however easy to remedy this.

We first observe that (if Haar measure is coveniently normalized)

$$
\int_{\hat{\sigma}} \phi_{1}(\xi(t)) \xi^{\prime}(t)^{m} \overline{\phi_{2}(\xi(s)) \xi^{\prime}(s)^{m}} d H(\xi)=K_{\beta}(t, s)\left(\phi_{1}, \phi_{2}\right)_{\beta}
$$

It follows that

$$
\begin{aligned}
& \int_{\hat{G}} \int_{\Delta} \phi_{1}(\xi(t)) \xi^{\prime}(t)^{m} \overline{\psi_{1}(t)} d \mu_{\beta}(t) \cdot \overline{\int_{\Delta} \phi_{2}(\xi(t)) \xi^{\prime}(t)^{m} \overline{\psi_{2}(t)}} d \mu_{\beta}(t) d H(\xi) \\
& =\left(\phi_{1}, \phi_{2}\right)_{\beta}{\overline{\left(\psi_{1}, \psi_{2}\right)_{\beta}}}_{\beta}
\end{aligned}
$$

If we apply the above to our case we find that

$$
\int_{\hat{G} \times\{1, \infty)}\left|F_{0}\left(z_{1}, z_{2}\right)\right|^{2} d H(\xi) d \Lambda_{\alpha}(\lambda)=\sum_{i} \int_{\hat{G}}\left|A_{i}(\xi)\right|^{2} d H(\xi)=\sum_{i, j}\left|p_{i j}\right|^{2}\|\phi\|_{\beta}^{2}
$$

However, in view of the definition of $a$,

$$
\int_{\Delta \times(1, \infty)}|a(t, \lambda)|^{2} d \mu_{\beta}(t) d \Lambda_{\alpha}(\lambda)=\sum_{i, j}\left|p_{i j}\right|^{2}
$$

Therefore, we conclude that formula (8) produces H.S. operators iff the last integral is finite. We have not investigated if it is possible to write this condition directly on $A$.

\section{6. $S_{p}$-norm}

What about the $S_{p}$-theory? Let us restruct the domain of definition of our operators to the smaller spaces $A^{\alpha, 2}(\Delta)$. An approach à la [A], [AFP], [BJP] then starts with two things: $1^{\circ}$ a direct computation of the $s$-numbers for a specially chosen symbol (viz. $b=z^{r}$ with a suitable $r$ ) and $2^{\circ}$ a direct treatment of the bounded operators. The rest is then more or less standard (duality, interpolation etc.). As for $1^{\circ}$, to make this approach work one has essentially to assume that $A\left(z_{1}, z_{2}, t\right)$ is homogeneous in its arguments. Then the operator $\left(T_{F}\right)^{*} T_{F}$ for $\phi \equiv 1$ is diagonal so everything blows down to computing an integral. We have not yet considered this question. 


\section{An infinitesimal approach, not entirely successful ${ }^{3}$}

Consider the group $G=S U(1,1) \approx S O(2,1)$. The corresponding Lie algebra $\mathrm{g}=\mathbf{s u}(1,1) \approx s o(2,1)$ is generated by the three matrices

$$
e_{3}=\left(\begin{array}{cc}
\frac{i}{2} & 0 \\
0 & -\frac{i}{2}
\end{array}\right), e_{1}=\left(\begin{array}{cc}
0 & \frac{1}{2} \\
\frac{1}{2} & 0
\end{array}\right), e_{2}=\left(\begin{array}{rr}
0 & \frac{i}{2} \\
-\frac{i}{2} & 0
\end{array}\right)
$$

corresponding to the one parameter semigroups

$$
\left(\begin{array}{cc}
e^{i \frac{\varepsilon}{2}} & 0 \\
0 & e^{-i \frac{\varepsilon}{2}}
\end{array}\right),\left(\begin{array}{cc}
\cosh \left(\frac{\epsilon}{2}\right) & \sinh \left(\frac{\varepsilon}{2}\right) \\
\sinh \left(\frac{\varepsilon}{2}\right) & \cosh \left(\frac{\varepsilon}{2}\right)
\end{array}\right),\left(\begin{array}{cc}
\cosh \left(\frac{\varepsilon}{2}\right) & i \sinh \left(\frac{\epsilon}{2}\right) \\
-i \sinh \left(\frac{\varepsilon}{2}\right) & \cosh \left(\frac{\epsilon}{2}\right)
\end{array}\right)
$$

Structure equations:

$$
\left[e_{1}, e_{2}\right]=-e_{3},\left\{e_{2}, e_{3}\right\}=e_{1},\left[e_{3}, e_{2}\right]=e_{3} .
$$

The complexified Lie algebra $\mathrm{g}_{\mathrm{c}}=\mathrm{sl}(2, \mathrm{C}) \approx \mathrm{so}(3, \mathrm{C})$ has generators

$$
h=-2 i e_{3}=\left(\begin{array}{rr}
1 & 0 \\
0 & -1
\end{array}\right), e=e_{1}-i e_{2}=\left(\begin{array}{ll}
0 & 1 \\
0 & 0
\end{array}\right), f=e_{1}+i e_{2}=\left(\begin{array}{ll}
0 & 0 \\
1 & 0
\end{array}\right) \text {. }
$$

Structure equations:

$$
[e, f]=h,[h, e]=2 e,[h, f]=-2 f .
$$

Casimir element:

$$
c=e_{1}^{2}+e_{2}^{2}-e_{3}^{2}=\frac{1}{4}\left(2(e f+f e)+h^{2}\right) .
$$

Let, as before, the group $G$ act on the Hilbert space $L^{2}\left(\Delta, \mu_{\alpha}\right)$ via the unitary maps

$$
U_{x}: f(z) \mapsto f(x(z))(c z+d)^{-\nu} \quad\left(x=\left(\begin{array}{ll}
a & b \\
c & d
\end{array}\right) \in G\right),
$$

where we now have put $\nu=\alpha+2$. Let also linear operators on $L^{2}\left(\Delta, \mu_{\alpha}\right)$ be given by kernels $F=F(z, w)$. On such functions, accordingly, $G$ operates via

$$
F(z, w) \mapsto F(x(z), x(w))(c z+d)^{-\nu}(\overline{c w+\bar{d}})^{-\nu} .
$$

Let $E_{1}, E_{2}, E_{3}$ be the infinitesimal operators corresponding to $e_{1}, e_{2}, e_{3}$. Considering the corresponding one parameter subgroups, one finds easily:

$$
\begin{aligned}
& E_{3} f=i\left(z \frac{\partial f}{\partial z}-\bar{z} \frac{\partial f}{\partial \bar{z}}+w \frac{\partial f}{\partial w}-\bar{w} \frac{\partial f}{\partial \bar{w}}\right), \\
& \left.E_{1} f=\frac{1}{2}\left(\left(1-z^{2}\right) \frac{\partial f}{\partial z}+\left(1-\bar{z}^{2}\right) \frac{\partial f}{\partial \bar{z}}+\left(1-w^{2}\right) \frac{\partial f}{\partial w}+\left(1-\bar{w}^{2}\right) \frac{\partial f}{\partial \bar{w}}-\nu z f-\nu \bar{w} f\right)\right), \\
& E_{2} f=\frac{i}{2}\left(\left(1+z^{2}\right) \frac{\partial f}{\partial z}-\left(1+\bar{z}^{2}\right) \frac{\partial f}{\partial \bar{z}}+\left(1+w^{2}\right) \frac{\partial f}{\partial w}-\left(1+\bar{w}^{2}\right) \frac{\partial f}{\partial \bar{w}}+\nu z f-\nu \bar{w} f\right) .
\end{aligned}
$$

\footnotetext{
${ }^{3}$ As most calculations of this Section have been checked on Mathematica, we allow us to omit many details.
} 
If, similarly, $E, F, H$ correspond to $e, f, h$, this shows that

$$
\begin{aligned}
& E=E_{\mathrm{I}}-i E_{2}=\frac{\partial}{\partial z}-\bar{z}^{2} \frac{\partial}{\partial \bar{z}}+\frac{\partial}{\partial w}-\bar{w}^{2} \frac{\partial}{\partial \bar{w}}-\nu \bar{w}, \\
& F=E_{1}+i E_{2}=-z^{2} \frac{\partial}{\partial z}+\frac{\partial}{\partial \bar{z}}-w^{2} \frac{\partial}{\partial w}+\frac{\partial}{\partial \bar{w}}-\nu z, \\
& H=2 i E_{3}=2\left(-z \frac{\partial}{\partial z}+\bar{z} \frac{\partial}{\partial \bar{z}}-w \frac{\partial}{\partial w}+\bar{w} \frac{\partial}{\partial \bar{w}}\right) .
\end{aligned}
$$

In order to decompose the action of $G$ on the kernels $F(z, w)$ into its irreducible constituents, we must solve the eigenvalue problem for the Casimir operator

$$
C=\frac{1}{2}(E F+F E)+\frac{1}{4} H^{2} .
$$

Write, for convenience,

$D=\frac{\partial}{\partial z}, \ddot{D}=\frac{\partial}{\partial \bar{z}}, T=\frac{\partial}{\partial w}, \bar{T}=\frac{\partial}{\partial \bar{w}} \quad(T$ for Esthonian "tuletis" ("derivative"))

Then the above formulae can be written

$$
\begin{aligned}
& E=D+T-\left(\bar{z}^{2} \bar{D}+\bar{w}^{2} T+\nu \bar{w}\right), \\
& F=\bar{D}+\bar{T}-\left(z^{2} D+w^{2} T+\nu z\right), \\
& H=-z D+\bar{z} \bar{D}-w T+\bar{w} \bar{T} .
\end{aligned}
$$

It follows that

$$
\begin{aligned}
& C=\left(1-|z|^{2}\right)^{2} D \bar{D}+\left(1-|w|^{2}\right)^{2} T \bar{T}+(1-z \bar{w})^{2} D \bar{T}+(1-\bar{z} w)^{2} \bar{D} T-(z-w)^{2} D T- \\
& (\bar{z}-\bar{w})^{2} \bar{D} \bar{T}-\nu\left[(1-z \bar{w}) z D+(1-z \bar{w}) \bar{w} T+\left(\bar{w}-|z|^{2} z\right) \bar{D}+\left(|w|^{2} w-z\right) T+1\right]+\nu^{2} z \bar{w} .
\end{aligned}
$$

We have not carried out the complete spectral analysis for this operator, but let us at lcast consider here some examples.

Example. (corresponding to big Hankel operators with non-analytic symbols; cf. Example 1 in Sec. 4). As before (Sec. 1) let $K_{\alpha}(z, w)=(1-z \ddot{w})^{-\nu}$ denote the reproducing kerncl in $A^{\alpha, 2}(\Delta)$. Take $F$ of the form

$$
F(z, w)=(\overline{b(z)-b(w)}) K_{\alpha}(z, w) .
$$

Then, after some miraculous simplifications, one obtains

$$
C F=\left(\overline{\left(1-|z|^{2}\right) D \bar{D} b(z)-\left(1-|w|^{2}\right) D T \bar{T} b(w)}\right) K_{\alpha}(z, w) .
$$

We recognize therein the usual $G$-invariant Laplace operator (cf: e.g. [AFP]). 
The above calculations become somewhat less miraculous if one first, quite generally, makes the substitution (cf. Sec. 2)

$$
F(z, w)=K_{\alpha}(z, w) F_{0}(z, w) .
$$

Then one sees that to the previous Casimir operator $C$ there corresponds the operator

$$
\begin{gathered}
C_{0}=\left(1-|z|^{2}\right)^{2} D \bar{D}+\left(1-|w|^{2}\right)^{2} T \bar{T}+ \\
+(1-z \bar{w})^{2}\left(D-\left(\frac{\bar{z}-\bar{w}}{1-z \bar{w}}\right)^{2} \bar{D}\right) \bar{T}+(1-z \bar{w})^{2}\left(T-\left(\frac{z-w}{1-z \bar{w}}\right)^{2} \bar{T}\right) D .
\end{gathered}
$$

In the next example we have in mind we restrict our attention to operators mapping the space $A^{\alpha, 2}(\Delta)$ into itself (operators of Toeplitz type), that is, we take $F(z, w)$ analytic in the first argument and anti-analytic in the second argument. Then some more interesting conclusions can be made so we relegate the resulting discussion to a separate division.

\section{Generalized Toeplitz operators}

If the kernel $F(z, w)$ is analytic in $z$ and conjugate-analytic in $w$ (analytic in $\bar{w}$ ) then the action of the Casimir operator $C$ on such functions reduces to

$$
C=(1-z \bar{w})^{2} D \bar{T}-\nu[(1-z \bar{w}) z D+(1-z \bar{w}) \bar{w} \bar{T}-1]+\nu^{2} z \bar{w} .
$$

To obtain the desired spectral decomposition it suffices to look at eigenfunctions $F$ which are rotation invariant, i.e. $F=f(z \bar{w})$. Then

$$
\begin{aligned}
& \frac{\partial F}{\partial z}=\bar{w} f^{\prime}(z \bar{w}) \Rightarrow z \frac{\partial F}{\partial z}=z D F=z \bar{w} f^{\prime}(z \bar{w}), \\
& \frac{\partial^{2} F}{\partial z \partial \bar{w}}=D \vec{T} F=z \bar{w} f^{\prime \prime}(z \bar{w})+f^{\prime}(z \bar{w}) .
\end{aligned}
$$

Thus we get the eigenvalue equation $(t=z \bar{w})$

$$
(1-t)^{2}\left(t f^{\prime \prime}+f^{\prime}\right)-2 \nu(1-t) t f^{\prime}+\nu^{2} t f=\lambda f .
$$

Introducing the notation $\mathcal{D}=t \frac{d}{d t}$, the operator ocurring here can also be written

$$
\left(t^{-1}+t-2\right) \mathcal{D}^{2}-2 \nu(1-t) \mathcal{D}+\nu^{2} t
$$

or again

$$
t^{-1} \mathcal{D}^{2}+t(\mathcal{D}+\nu)^{2}-\mathcal{D}(\mathcal{D}+\nu)
$$


Thus, the eigenfunctions are of the form

$$
F(z, w)=\sum_{n=0}^{\infty} p_{n}(\lambda) t^{n} \quad(t=z \bar{w})
$$

where the $p_{n}(\lambda)$ are certain orthogonal polynomials determined by the recursion

$$
(n+1)^{2} p_{n+1}+(n+\nu-1)^{2}-2 n(n+\nu) p_{n}=\lambda p_{n} .
$$

These orthogonal polynomials are mentioned in [P4], but they are special cases of polynomials considered by Askey and Wilson (see e.g. [W]). ${ }^{4}$ (It would be interesting to find out what the the corresponding orthogonal functions are in the case of, say, the ball, cf. Introduction.) It follows from their formulae that the spectral measure is concentrated on the interval $\left[\frac{1}{4}, \infty\right)$. From this and known facts (see e.g. [AFP]) about the decomposition of the group action in $L^{2}(\Delta, I)(I=$ Poincaré measure), one can draw an interesting conclusion: The Hilbert space of Hilbert-Schmidt operators on $A^{\alpha, 2}(\Delta)$ and the Hilbert space $L^{2}(\Delta, I)$, both considered as unitary $G$-modules, are isomorphic. (In itself, such as result is not new, see e.g. $[R]^{5}$.) That is, there exists an "operator calculus", a unitary map $L^{2}(\Delta, I) \rightarrow S^{2}\left(A^{\alpha, 2}(\Delta)\right): h \mapsto W(h)$ which intertwines with $G$ :

$$
U_{x} W(h) U_{x}^{-1}=W(h \circ x) \quad\left(x \in G, h \in L^{2}(\Delta, I) .\right.
$$

It would be of interest to compare this "abstract" calculus with the "concrete" calculi described in [UU].

\section{Appendix. Comparison of two invariant measures}

Consider any geodesic line in $\Delta\left(=\operatorname{arc}\right.$ of an orthogonal circle). Let $\zeta_{1}$ and $\zeta_{2}$ be its endpoints on the unit circumference $\mathbf{T}$. The general point $z$ on it can be written

$$
z=\frac{\zeta_{1}-\zeta_{2} i k m}{1-i k m}
$$

where $k^{2}=\frac{\zeta_{1}}{\zeta_{2}}, \operatorname{Im} k>0$ and $m$ is the logarithmic distance from $z$ to the Euclidean midpoint $z^{*}$ of the circular arc. ${ }^{6}$ Then

$$
d z=\frac{d \zeta_{1}-d \zeta_{2} i k m}{1-i k m}+\frac{\left(\zeta_{1}-\zeta_{2}\right) i d(k m)}{(1-i k m)^{2}} \equiv A d \zeta_{1}+B d \zeta_{2}+C d m
$$

\footnotetext{
${ }^{4}$ I was told about this by $R$. Askey.

${ }^{5}$ I owe this piece of information, and the reference to $A$. Unterberger (personal communication). ${ }^{6}$ Alternatively, $z^{*}$ is the intersection with the geodesic through the origin which cuts the given one perpendicularly?
} 
where (use that $2 \frac{d k}{k}=\frac{d \zeta_{1}}{\zeta_{1}}-\frac{d \zeta_{2}}{\zeta_{2}}$ )

$$
\begin{aligned}
A & =\frac{1-i m \operatorname{Re} k}{(1-i k m)^{2}}, \\
B & =\frac{(i k m)^{2}\left(1+i m^{-1} \mathrm{Re} k\right)}{(1-i k m)^{2}}, \\
C & =\frac{\left(\zeta_{1}-\zeta_{2}\right) i k}{(1-i k m)^{2}} .
\end{aligned}
$$

Modulo terms of degree 2 in $d \zeta_{1}$ and $d \zeta_{2}$ we have

$$
\begin{gathered}
d z d \bar{z}=\left(A d \zeta_{1}+B d \zeta_{2}\right) \bar{C} d m-\left(\bar{A} d \bar{\zeta}_{1}+\bar{B} d \bar{\zeta}_{2}\right) C d m+O(2) \\
=\left(\left(A \bar{C} d \zeta_{1}-\bar{A} C d \bar{\zeta}_{1}\right)+\left(B \bar{C} d \zeta_{2}-\bar{B} C d \bar{\zeta}_{2}\right)\right) d m+O(2) \\
\equiv\left(M d \zeta_{1}+N d \zeta_{2}\right) d m+O(2)
\end{gathered}
$$

where $\left(\operatorname{as} \bar{\zeta}=\frac{1}{\zeta} \Rightarrow d \bar{\zeta}=-\frac{d \zeta}{\zeta^{2}}\right)$

$$
M=A \bar{C}-\bar{A} C \times\left(-\frac{1}{\zeta_{1}^{2}}\right), \quad N=B \bar{C}-\bar{B} C \times\left(-\frac{1}{\zeta_{2}^{2}}\right) .
$$

If $z_{1}$ and $z_{2}$ are two variable points on the geodesic then

$$
\begin{aligned}
d z_{1} d \bar{z}_{1} d z_{2} d \bar{z}_{2} & =-\left(M_{1} d \zeta_{1}+N_{1} d \zeta_{2}\right)\left(M_{2} d \zeta_{1}+N_{2} d \zeta_{2}\right) d m_{1} d m_{2}+O(3) \\
= & -\left(M_{1} N_{2}-M_{2} N_{1}\right) d \zeta_{1} d \zeta_{2} d m_{1} d m_{2}+O(3) .
\end{aligned}
$$

We have

$$
\begin{gathered}
M=\frac{1}{|1-i k m|^{4}}\left\{(1-i m \operatorname{Re} k)\left(\overline{\zeta_{1}-\zeta_{2}}\right)(-i \bar{k})-(1+i m \operatorname{Re} k)\left(\zeta_{1}-\zeta_{2}\right) i k\left(-\frac{1}{\zeta_{1}^{2}}\right)\right\} \\
=\frac{2 i k\left(\zeta_{1}-\zeta_{2}\right)}{|1-i k m|^{4} \zeta_{1}^{2}}, \\
N=\frac{1}{|1-i k m|^{4}}\left\{(i k m)^{2}\left(1+i m^{-1} \operatorname{Re} k\right)\left(\bar{\zeta}_{1}-\bar{\zeta}_{2}\right)(-i \bar{k})-\right. \\
\left.\left(-i \bar{k} m^{2}\right)\left(1-i m^{-1} \operatorname{Re} k\right)\left(\zeta_{1}-\zeta_{2}\right) i k\left(-\frac{1}{\zeta_{2}^{2}}\right)\right\}=\frac{2(i k)^{3} m^{2}\left(\zeta_{1}-\zeta_{2}\right)}{|1-i k m|^{4} \zeta_{1}^{2}} .
\end{gathered}
$$

Thus

$$
M_{1} N_{2}-M_{2} N_{1}=\frac{4(i k)^{4}\left(\zeta_{1}-\zeta_{2}\right)^{2}\left(m_{2}^{2}-m_{1}^{2}\right)}{\left|1-i k m_{1}\right|^{4}\left|1-i k m_{2}\right|^{4} \zeta_{1}^{4}} \stackrel{\text { sice! }}{=} \frac{4\left|\zeta_{1}-\zeta_{2}\right|^{2}\left(m_{2}^{2}-m_{1}^{2}\right)}{\left|1-i k m_{1}\right|^{4}\left|I-i k m_{2}\right|^{4} \zeta_{1} \zeta_{2}} .
$$


On the other hand

$$
\begin{gathered}
|z|^{2}=\frac{\left|\zeta_{1}-\zeta_{2} i k m\right|^{2}}{|1-i k m|^{2}}=\frac{1+|k|^{2} m^{2}-2 \operatorname{Re} i k m \overline{\zeta_{1}} \zeta_{2}}{1+k^{2} m^{2}-2 \operatorname{Re} i k m}, \\
1-|z|^{2}=\frac{2 \operatorname{Re}\left(i k m\left(\bar{\zeta}_{1} \zeta_{2}-1\right)\right)}{|1-i k m|^{2}} .
\end{gathered}
$$

$\mathrm{But}\left(\operatorname{as} \bar{k}=\frac{k \zeta_{2}}{\zeta_{1}}\right)$

$$
i k\left(\frac{\zeta_{2}}{\zeta_{1}}-1\right)-i \bar{k}\left(\frac{\zeta_{1}}{\zeta_{2}}-1\right)=i k\left(\zeta_{2}-\zeta_{1}\right)\left(\frac{1}{\zeta_{1}}+\frac{\zeta_{2}}{\zeta_{1}} \frac{1}{\zeta_{2}}\right)=\frac{2 i k\left(\zeta_{2}-\zeta_{1}\right)}{\zeta_{1}} .
$$

Thus

$$
\left(1-|z|^{2}\right)^{2}=\frac{4\left|\zeta_{1}-\zeta_{2}\right|^{2} m^{2}}{|1-i k m|^{4}}
$$

and

$$
\left(1-\left|z_{1}\right|^{2}\right)^{2}\left(1-\left|z_{2}\right|^{2}\right)^{2}=\frac{16\left|\zeta_{1}-\zeta_{2}\right|^{4} m_{1}^{2} m_{2}^{2}}{\left|1-i k m_{1}\right|^{4}\left|1-i k m_{2}\right|^{4}}
$$

It follows that

$$
\left(\frac{i}{2 \pi}\right)^{2} \times \frac{d z_{1} d \bar{z}_{1} d z_{2} d \bar{z}_{2}}{\left(1-\left|z_{1}\right|^{2}\right)^{2}\left(1-\left|z_{2}\right|^{2}\right)^{2}}=\frac{1}{16 \pi^{2}} \frac{m_{1}^{2}-m_{2}^{2}}{m_{1} m_{2}} \frac{\left|d \zeta_{1}\right| \cdot\left|d \zeta_{2}\right|}{\left|\zeta_{1}-\zeta_{2}\right|^{2}} \frac{d m_{1}}{m_{1}} \frac{d m_{2}}{m_{2}}
$$

as previously claimed (Sec. 2, formula (6)).

\section{References}

[A] S. AxlER, The Bergman space, the Bloch space, and commutators of multiplication operators, Duke Math. J. 53 (1986), 315-332.

[AFP] J. ARAZY, S. Fisher AND J. PEeTre, Hankel operators on weighted Bergman spaces, Amer. Math. J. 110 (1988), 989-1053.

[BJP] J. Boman, S. Janson AND J. PEeTre, Big Hankel operators of higher weight, Rend. Circ. Mat. Palermo 37 (1988), 65-78.

[GP] B. Gustafsson and 3. PeEtre, Notes on projective structures on complex manifolds, Nagoya Math. J. 116 (1989), 63-88.

[JP1] S. JANSON AND J. PEETRE, A new generalization of Hankel operators (the case of higher weights), Math. Nachr. 132 (1987), 313-328.

[JP2] S. Janson AND J. PEetre, Paracommutators - boundedness and Schatten-von Neumann properties, Trans. Am. Math. Soc. 305 (1988), $467-504$. 
[JPR] S. Janson, J. Peetre And R. Rocherg, Hankel forms and the Fock space, Revista Mat. Ibero.Amer. 3 (1987), 61-138.

[JPW] S. Janson, J. PeEtre and R. Wallstén, A new look on Hankel forms over Fock space, Studia Math. 95 (1989), 33-41.

$[\mathrm{L}]$ S. LANG, " $S L_{2}(\mathbf{R})$," Addison-Wesley Publishing Co., Reading, Mass. London - Amsterdam, 1975.

[P1] J. PEETRE, "Hankel kernels of higher weight for the ball," Technical report, Lund, 1988.

[P2] J. PEETRE, Paracommutators - a brief introduction, open problems, Revista Mat. Univ. Complutense Madrid 2, número suplementario (to appear).

[P3] J. PeETrE, The Berezin transform and Ha-plitz operators, J. Operator Theory (to appear).

[P4] J. PeEtre, Some unsolved problems, In: Colloquie Mathematica Societatis Janos Bolyai, Alfred Haar Memorial Conference, Budapest (Hungary), 711-735, 1985. North-Holland, Amsterdam, 1986.

[R] G. RiDEAU, Sur la réduction du produit tensoriel des répresentations de la série discrète de $S L(2, \mathbf{R})$, Ann. Inst. H. Poincaré Sect. A. 4 (1966), $67-76$.

[UU] A. Unterberger and J. Unterberger, La série discrète de $S L(2, \mathbf{R})$ et les opérateurs pseudo-différentiels sur une semi-droite, Ann. Sci. Ecole Norm. Sup. 17 (1984), 83-116.

[W] J. WILSON, Some hypergeometric orthogonal polynomials, SIAM J. Math. Anal. 11 (1980), 690-701.

Keywords. Hankel operator, reproducing kernel, decomposition of group representations, Hilbert-Schmidt operator

1980 Mathematics subject classifications: 47B35, 30C40, 43A65, 43A80

Maternatiska Institutionen

Stockholms universitet

Box 6701

S-113 85 Stockholm

SWEDEN

Rebut el 16 de Juny de 1989 